\title{
Avances en la patogénesis de la embriopatía diabética
}

\author{
Damarys García G ${ }^{1}$, Ricardo G arcía D². \\ Recent advances in the pathogenesis \\ of diabetic embryopathy
}

The congenital malformations in the offspring of diabetic mothers are the result of a multifactorial process. Susceptibility to the effects of maternal diabetes in the pathogenesis of these anormalies is influenced by the genetic background, indicating that there are polymorphic genes that modify the cellular response to hyperglycemia. The modifier genes for the teratogenic effect of maternal diabetes are yet unknown. An excessive glucose supply to embryonic tissues leads to a state of oxidative stress, which affects the expression of genes encoding scavenging enzymes such as superoxide dismutase (SOD) and catalase and activates development genes such as PAX3, involved in neural tube defects. Cell proliferation and cell death are important mechanisms underlying malformations in infants born to diabetic mothers. There is an increase of apoptotic Bax and caspase 3 proteins and a low expression of Bcl-2 antiapoptotic protein in embryos exposed to a diabetic environment. Hyperglycemia decreases intracellular levels of reduced GSH, prostaglandin E2 (PGE2) and DNA synthesis in embryo's tissues. Understanding the molecular pathogenesis of diabetic embryopathy will allow the use of effective therapies for the prevention of teratogenic effects in diabetic mothers (Rev Méd Chile 2009; 137: 1627-35).

(Key w ords: Diabetes mellitus; Fetal diseases; Teratogens)

Recibido el 2 de mayo, 2008. Aceptado en versión corregida el 27 de enero, 2009.

${ }^{1}$ Centro Nacional de Genética Médica, Cuba. ${ }^{2}$ Instituto Nacional de Salud Ocupacional, Cuba.

L a a diabetes ha sido reconocida como una enfermedad que incrementa el riesgo de aparición de defectos congénitos (DC) en la descendencia de mujeres que la padecen de 3 a 5 veces en comparación con gestantes que no presentan la afección ${ }^{1-5}$.

Alrededor de $1 \%$ de todas las embarazadas presenta diabetes antes de la gestación y hasta en $12 \%$ se descubre este proceso en el curso del embarazo $^{6}$. La incidencia del efecto teratogénico no se limita a la diabetes tipo I, está descrita en la

Correspondencia a: Dr. Ricardo García D. Apartado Postal 9070. Zona Postal 9. Código Postal 10900. Ciudad Habana, Cuba. E mail: richardg@infomed.sld.cu diabetes tipo II y en la diabetes gestacional. Además, la obesidad materna puede incrementar el riesgo de anomalías congénitas de la misma forma que una diabetes franca? ${ }^{7}$.

La descompensación de la enfermedad alrededor del período de organogénesis ha sido relacionada con un espectro de alteraciones del desarrollo que incluye: anomalías del sistema nervioso, cardiovascular, renal, sistema esquelético, retardo en el crecimiento y aborto. Virtualmente cualquier órgano puede ser afectado ${ }^{8} \mathrm{y}$ entre $8 \%$ y $12 \%$ de las embarazadas diabéticas portan fetos con algún tipo de malformación ${ }^{9}$.

Los hijos de madres diabéticas al año de edad muestran puntajes mentales y psicomotores más bajos que los controles. De esta manera, la 
diabetes materna puede causar anomalías groseras de la morfología normal en algunos sistemas orgánicos y probablemente también sutiles defectos funcionales del SNC incluso en gestantes con tratamiento insulínico y adecuado control glicémico durante la gestación 10,11 .

Los principales hallazgos comunicados en los hijos de mujeres diabéticas incluyen defectos cardiovasculares y del sistema nervioso central como $^{13}$ :

Sistema cardiovascular. Transposición de los grandes vasos con o sin defectos del septum ventricular, defectos del septum ventricular, coartación de la aorta, hipoplasia del corazón izquierdo, defectos del septum auricular, tetralogía de Fallot, estenosis pulmonar.

Sistema nervioso central. Defectos del tubo neural. Anencefalia con o sin hernias de elementos neurales, hidrocefalia, hidranencefalia, microcefalia, espina bífida.

La producción de dichos defectos congénitos en la descendencia de madres diabéticas obedece a cinco cambios metabólicos fundamentales relacionados con el exceso de glucosa en el producto de la concepción, los cuales abordaremos en este trabajo, ellos son:

1. Expresión disminuida en los tejidos embrionarios de genes del desarrollo como el PAX3. 2. Disminución de las defensas antioxidantes del embrión. 3. Disminución de los niveles de prostaglandina E2 en el embrión. 4. Incremento de la apoptosis inducida por estrés oxidativo en los tejidos embrionarios. 5. Alteraciones en el funcionamiento del tejido placentario.

Se ha investigado que la hiperglicemia materna es uno de los principales factores en la producción de dichas malformaciones. El estudio de la patogénesis de los $\mathrm{DC}$, realizado en animales de experimentación, ha revelado un complejo proceso en el cual el estado diabético induce alteraciones en una serie de vías metabólicas interrelacionadas que conducen a la teratogenicidad al interferir la expresión de importantes genes del desarrollo embrionario ${ }^{12}$.

La oxidación de glucosa en exceso afecta la expresión de genes del desarrollo como el PAX3. Datos clínicos y experimentales indican que existe más de una alteración en el ambiente del embrión de madre diabética capaz de inducir un desarrollo anómalo, y probablemente la alteración más importante es el incremento de la concentración de glucosa $^{14}$ que tiene numerosas consecuencias metabólicas en el embrión ${ }^{7,15}$. Antes de la organogénesis el embrión avascular es fisiológicamente hipóxico $\left(2 \%-5 \% \mathrm{O}_{2}\right)$ comparado con la circulación materna $\left(20 \% \mathrm{O}_{2}\right)$. El incremento de la masa celular causa un estado fisiológico de hipoxia que lleva a la activación de genes que inducen la formación del sistema circulatorio fetal.

En esta etapa del desarrollo el embrión expresa genes de transportadores de glucosa de alta $\mathrm{Km}$ como el Glut2, y transportadores de baja $\mathrm{Km}$ como Glut1, Glut3 y posiblemente Glut8, lo cual no trae consecuencias adversas en el curso de un embarazo no diabético, pero sí puede explicar que las concentraciones intracelulares de glucosa en el embrión estén en equilibrio con las cifras séricas maternas y alcancen niveles 2-3 veces superiores en el embrión de madre diabética.

Según recientes investigaciones de Loeken MR y cols, existe un umbral de glicemia materna $\geq 250$ $\mathrm{mg} / \mathrm{dl}(13,89 \mathrm{mmol} / \mathrm{l})$ por encima del cual hay un incremento significativo en los defectos del tubo neural (DTN). Estas cifras se han podido detectar antes de la expresión del gen Pax3 y corresponde a la Km para el transportador Glut2, por lo que se considera que el transporte de glucosa mediado por Glut2 durante la organogénesis es responsable de los efectos embriopáticos de la diabetes materna ${ }^{16}$.

El aporte incrementado de glucosa a los tejidos embrionarios antes de la formación del sistema circulatorio, hace que aumente su metabolismo oxidativo con incremento del consumo de $\mathrm{O}_{2}$. La hipoxia generada excede los límites fisiológicos que caracterizan al normal desarrollo. En estas condiciones se puede estimular la producción mitocondrial de radical superóxido llevando a un estado de estrés oxidativo (EO) como consecuencia de la oxidación de la glucosa en exceso ${ }^{16,17}$.

Muchas investigaciones han demostrado que el EO producido por el exceso de glucosa tiene efecto embriotóxico ${ }^{14-23}$. Se ha propuesto que el EO provoca alteraciones en la activación sincrónica de genes importantes en el control de la morfogénesis temprana y por tanto, los DC se producen por un mecanismo diferente al de 
inducción de la muerte celular directa por daño oxidativo al material genético, rotura de las cadenas del ADN y apoptosis ${ }^{16}$.

Por otro lado la formación de productos avanzados de la glicosilación (AGE) puede causar roturas de simple cadena en el ADN genómico, que pueden tener serios efectos teratogénicos. Dichos efectos pueden también ser estimulados por la glicosilación de las proteínas histonas, que tienen un rol estructural vital en el mantenimiento de los nucleosomas y por consiguiente, en la integridad del ADN. Los azúcares intracelulares como la glucosa-6-fosfato y la ADP-ribosa reaccionan fuertemente con grupos aminoácidos de las histonas, donde pueden causar enlaces cruzados in vitro así como in vivo ${ }^{10}$.

En etapas tempranas del desarrollo la glucosa puede dañar el ADN y provocar mutaciones que impidan la expresión de genes críticos para la embriogénesis normal. Este daño puede retrasar la duplicación del ADN y, por lo tanto, la división celular, lo que interrumpe los tiempos de los programas del desarrollo necesario para la organogénesis. De hecho, aun la hiperglicemia moderada durante un periodo corto es capaz de producir mutaciones al ADN.

Se ha reconocido que el control del ciclo celular está muy relacionado con el metabolismo interme- diario y que la expresión de genes del desarrollo como el PAX3 está regulada por la actividad metabólica y el estado redox de la célula ${ }^{24}$.

El incremento de las especies reactivas del oxígeno (ERO) producido durante las reacciones de la cadena respiratoria mitocondrial y la fosforilación oxidativa llevan a una inhibición de la gliceraldehído fosfato deshidrogenasa (GAPDH), que actúa como coactivador transcripcional del ciclo celular y puede ser necesario para la inducción de la transcripción del gen PAX3 ${ }^{17}$.

El gen PAX3 se expresa en el neuroepitelio, crestas neurales y mesodermo somítico; su rol esencial en el cierre del tubo neural es inhibir la apoptosis mediada por p53 al mantener la regulación negativa de este último ${ }^{6,25}$.

Este factor de transcripción puede regular además la expresión del factor cdc46 Hill AL, y el gen Dep1. Ambos genes tienen importantes funciones en el cierre del tubo neural ${ }^{26}$. En el embrión de madre diabética el EO producido por las concentraciones elevadas de glucosa conduce a una inhibición de la expresión de PAX3 que lleva a desrepresión del gen p53 y muerte celular en el neuroepitelio, lo que conduce a los defectos del tubo neural (DTN) ${ }^{25}$ (Figura 1).

Gareskog $\mathrm{M}$ ha comprobado en un estudio experimental que la suplementación con ácido

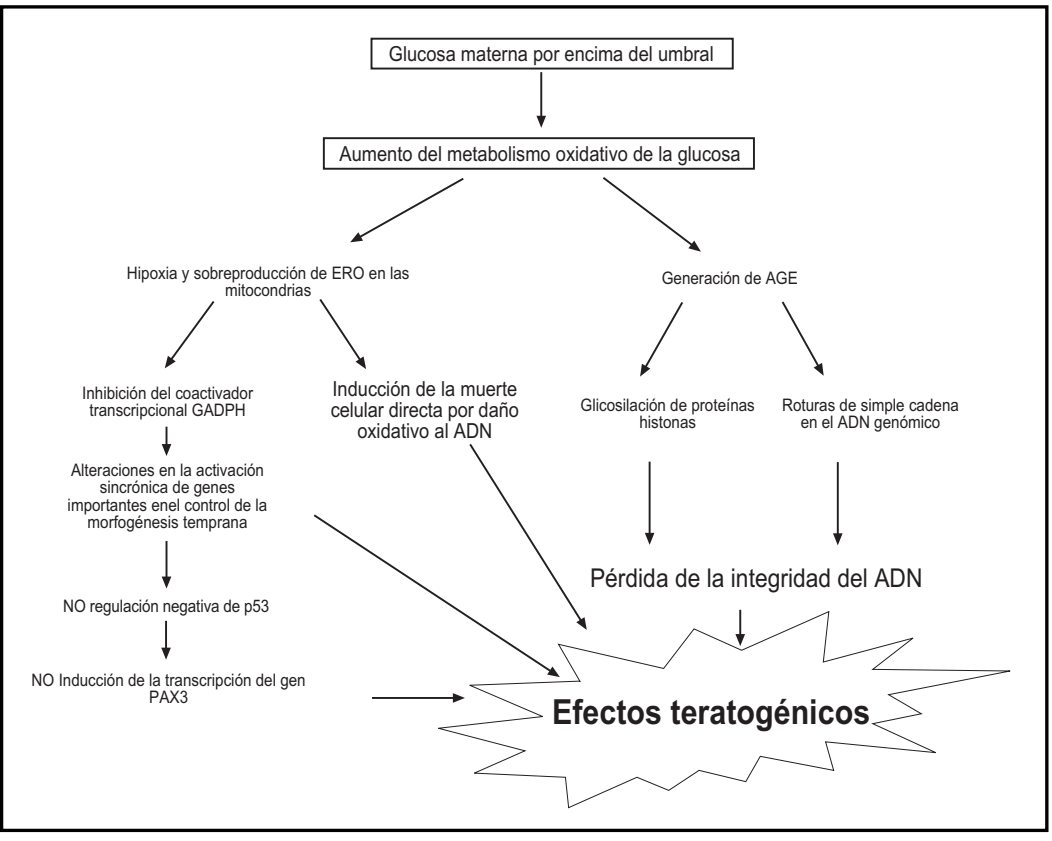

Figura 1. La oxidación de glucosa en exceso afecta la integridad del ADN y la expresión del gen PAX3.

ERO: especies reactivas del oxígeno. AGE: productos avanzados de la glicosilación. GAPDH: gliceraldehído fosfato deshidrogenasa. ADN: ácido desoxirribonucleico. 
fólico disminuye la dismorfogénesis en embriones de ratas expuestos a un ambiente hiperglicémico. Dicho experimento demuestra que existe un incremento de las demandas de ácido fólico, considerado un antioxidante, en el organismo expuesto al medio diabético. Otros estudios han demostrado que las defensas antioxidantes en general son deficientes en el embrión de madre diabética.

El exceso de glucosa provoca disminución en las defensas antioxidantes del embrión. El glutatión reducido (GSH) constituye un importante antioxidante a nivel celular, interviene en la síntesis de ADN y proteínas, y contribuye a la integridad de las membranas celulares ${ }^{27}$, la diabetes debilita el sistema antioxidante al afectar la enzima que cataliza su síntesis, la gammaglutamil-cisteína sintetasa (gamma-GCS), lo que lleva a una reducción de la concentración de GSH. La disminución de GSH y el incremento de las ERO alteran el desarrollo del embrión ${ }^{28}$.

La activación de la vía de la hexosamina producida por la hiperglicemia contribuye al deterioro de las defensas antioxidantes y al desarrollo de defectos congénitos en el embrión.

En la vía biosintética de la hexosamina (HBP) la fructosa-6-fosfato generada en la glicólisis sufre amidación por acción de la enzima glutamina-fructosa-6fosfato amidotransferasa (GFAT) generándose glucosamina-6-fosfato que actúa como inhibidor competitivo de la glucosa-6-fosfato deshidrogenasa (G6PD), enzima limitante del ciclo de las pentosas ${ }^{29}$. Durante el peńodo temprano de posimplantación, aproximadamente $25 \%$ de la glucosa es metabolizada a través del ciclo de las pentosas. Además de la generación de ribosa para la síntesis de nucleótidos, la vía de las pentosas produce NADPH a partir de $\mathrm{NADP}^{+}$. El NADPH sirve como cofactor para la glutatión reductasa que convierte el glutatión oxidado (GSSG) en glutatión reducido (GSH) ${ }^{6}$.

El exceso de glucosa en los tejidos fetales activa la vía del poliol en la cual la glucosa se reduce a sorbitol por la aldosa reductasa con consumo de NADPH. La actividad incrementada en esta vía en el medio diabético causa una depleción del glutatión reducido por déficit de NADPH ${ }^{16}$ (Figura 2).

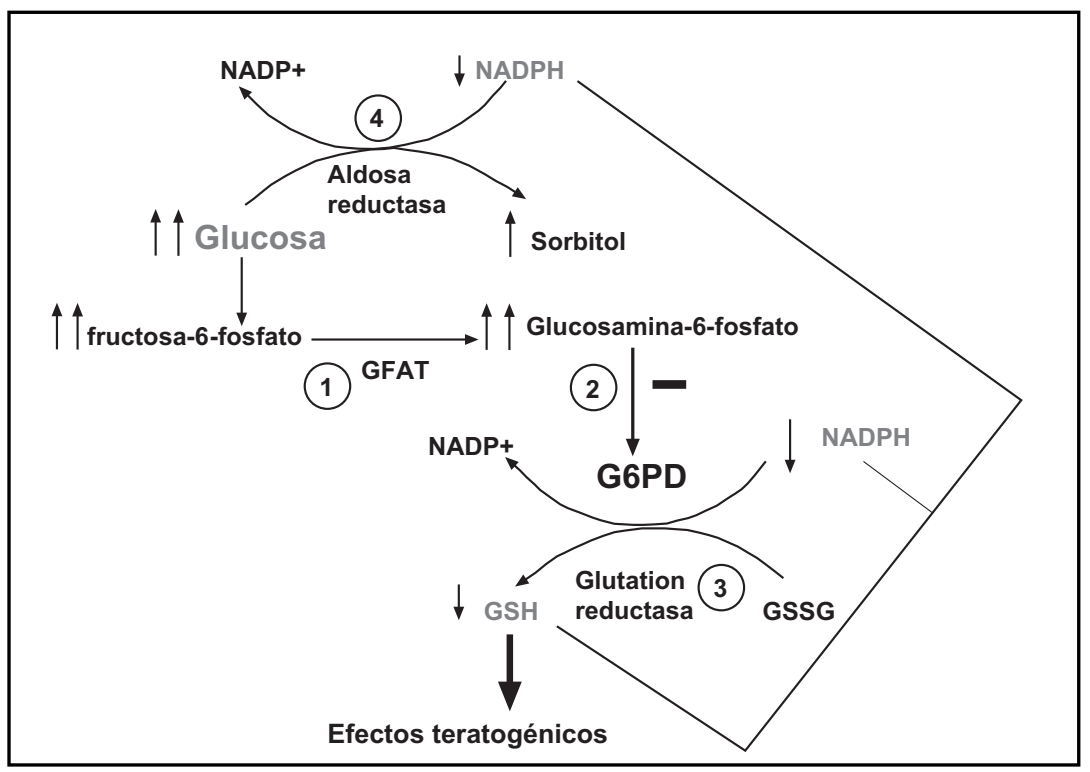

Figura 2. Disminución de las defensas antioxidantes en el embrión de madre diabética.

1) La enzima glutamina-fructosa-6-fosfato amidotransferasa (GFAT) transforma la fructosa-6-fosfato en glucosamina-6fosfato en la vía biosintética de la hexosamina (HBP). 2) La glucosamina-6-fosfato actúa como inhibidor competitivo de la glucosa-6-fosfato deshidrogenasa (G6PD), enzima limitante del ciclo de las pentosas. 3) La glutatión reductasa convierte el glutatión oxidado (GSSG) en glutatión reducido (GSH) con consumo de NADPH producido en la vía de las pentosas. 4) GLucosa en exceso en los tejidos fetales se reduce a sorbitol por la acción de la aldosa reductasa, el consumo de NADPH en esta reacción causa una depleción del glutatión reducido por déficit de NADPH. 
Se ha demostrado que la expresión de los genes que codifican para enzimas antioxidantes se afecta en la diabetes.

El hecho de que cepas de embriones de ratas resistentes a los defectos congénitos expresen niveles incrementados de ARNm de $\mathrm{Mn}$-superóxido dismutasa (SOD) y catalasa mientras que en embriones malformados no se incrementa la transcripción de estos genes sugiere que los altos niveles de enzimas antioxidantes protege a los embriones del estrés oxidativo causado por la diabetes materna ${ }^{21,30,31}$.

La disminución de las defensas antioxidantes en el embrión, el EO y el incremento de la peroxidación lipídica que se produce como consecuencia conducen a un incremento de la muerte celular programada (apoptosis) que es considerada como uno de los mecanismos de producción de las defectos congénitos en la gestación diabéti$\mathrm{ca}^{32-34}$.

Papel de la apoptosis inducida por EO en la producción de los defectos congénitos. Se ha ob- servado que la diabetes materna causa disminución de la actividad del factor nuclear kappa B (NF-kappaB) y de las concentraciones de la proteína B-cell lymphoma 2 (Bcl-2), e incremento de la activación de la proteína caspasa 3 (un componente esencial de múltiples vías apoptóticas) y de los niveles de proteínas Bax agonistas de la apoptosis ${ }^{35}$.

El NF-kappa B en particular, está implicado en el control del crecimiento, reacciones inflamatorias y la apoptosis, y es el primer factor de transcripción eucarionte que ha mostrado responder directamente al EO ${ }^{27}$. La activación de NFkappa B se produce a través del receptor de superficie celular del factor de necrosis tumoral 1 (TNFR1).

El TNFR1 está relacionado con la proliferación celular y la apoptosis en respuesta a señales del ambiente intra y extracelular como proteínas citoplasmáticas, factores de crecimiento, agentes químicos mutágenos, radiaciones, etc. La activación de TNFR1 se produce también por incremento de la densidad de receptores en la superficie

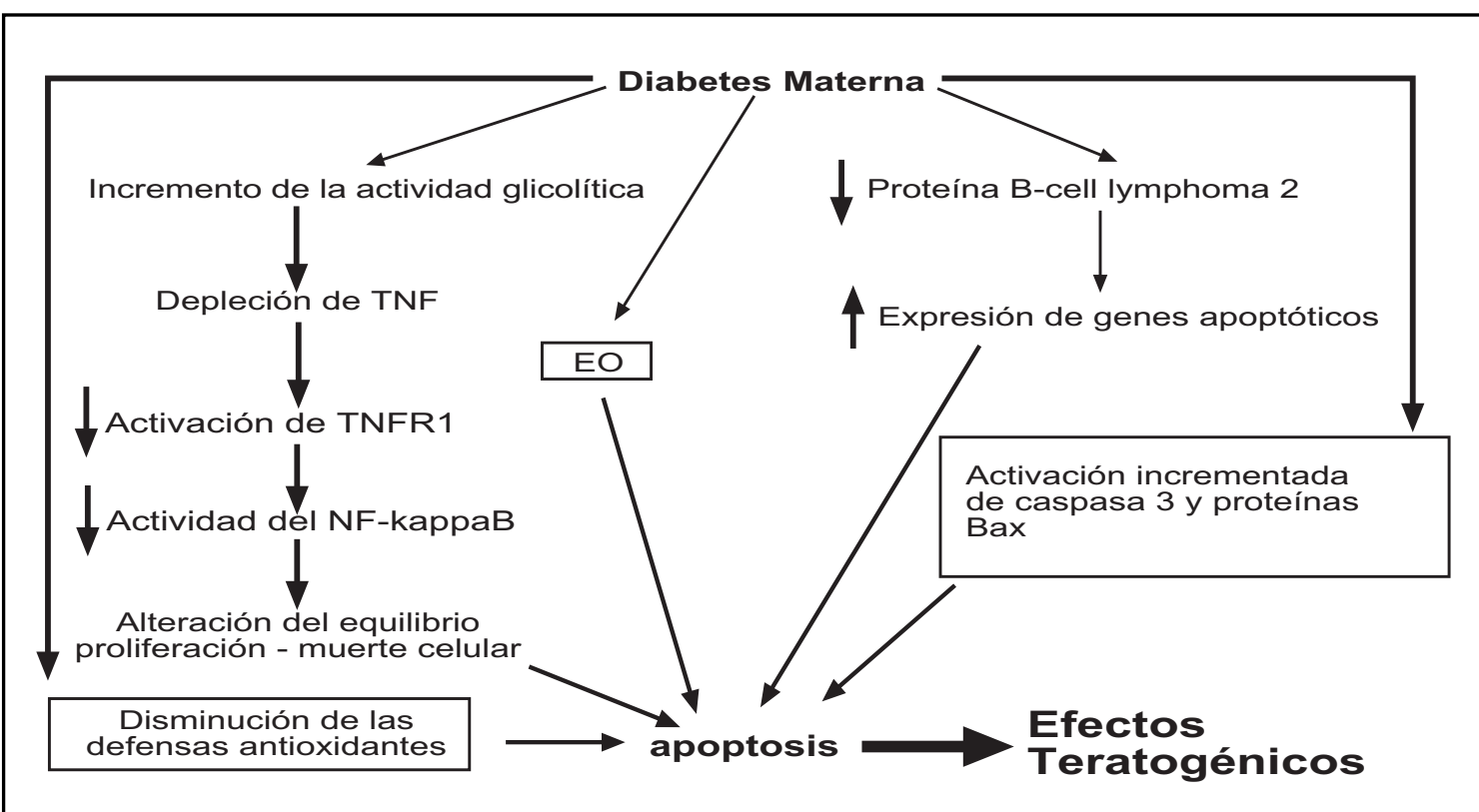

Figura 3. La diabetes materna causa apoptosis y efectos teratogénicos en el embrión. EO: estrés oxidativo. NF-kappa B: factor nuclear kappa B. TNFR1: receptor de superficie celular del factor de necrosis tumoral 1. TNF: factor de necrosis tumoral. 
celular y la depleción de TNF ha sido mediada, en cierta manera, por el incremento de la actividad glicolítica y de la producción de lactato inducido por TNF.

El TNFR1 puede tener actividad antiapoptótica cuando su activación se produce a través de proteínas citoplasmáticas con dominios DD (death domins), cuya unión al receptor desencadena una serie de reacciones en cascada que conducen a la proliferación celular y no a la muerte de la célula. En cambio, si la proteína ligando que se une al dominio $\mathrm{N}$ terminal del receptor TNF posee dominios DED (death effectors domins), resulta más probable que las reacciones consecutivas conduzcan a la muerte celular programada.

Un adecuado equilibrio de los mecanismos de proliferación y muerte celular es imprescindible para el normal desarrollo del embrión ${ }^{36}$.

Por otro lado, la familia de proteínas bcl2 puede proteger a la célula de la apoptosis. Se han localizado en las membranas citoplasmática y nuclear donde se cree que su función es la formación de poros. Se le ha atribuido la función de regular negativamente la expresión de genes que codifican productos apoptóticos y se conoce que previene la salida de citocromo $\mathrm{C}$ de la mitocondria, lo cual es un evento esencial en la muerte celular ${ }^{37}$. Al menos 11 genes relacionados con bcl2 han sido identificados en mamíferos e incluyen antagonistas de la apoptosis (bcl2, bclx1, bcl-w, bfl-1, BRAG1 y mcl-1) e inductores de la apoptosis (bax, bik, bad y bcl-xs) ${ }^{38}$.

Se ha hipotetizado que las caspasas pueden alterar la especificidad de sustrato de las quinasas, o que las quinasas pudieran fosforilar sitios de clivaje en los sustratos de las caspasas, regulando de esta manera su actividad 36 .

En embriones de ratas diabéticas se ha detectado incremento de la actividad de la proteína quinasa C-alpha (PKC-alpha), PKC-beta1, PKCgamma, PKC-delta, y PKC-zeta en el día 10 de la gestación. Por el contrario, el día 11 de la gestación se ha detectado una disminución de los niveles de PKC-alpha y PKC-zeta y se ha observado asociación entre niveles aumentados de actividad de PKC-gamma y PKC-delta, y la aparición de defectos congénitos. Otros estudios sugieren que una disminución de la actividad de PKC se relaciona con la aparición de defectos en la descendencia de gestante diabética, lo que indica que se requiere una actividad basal de PKC para el normal desarrollo embrionario. Aún se necesitan investigaciones adicionales para esclarecer el papel de PKC en la etiología de la embriopatía diabética ${ }^{39,40}$.

La suplementación combinada con vitamina $\mathrm{E}$ y ácido fólico en ratas diabéticas embarazadas disminuye las resorciones de los tejidos y normaliza los niveles de proteínas apoptóticas, aunque no se logra eliminar completamente los daños fetales, lo que apoya la idea de que otros mecanismos además del EO y la apoptosis están involucrados en la embriopatía diabética ${ }^{31}$.

Al respecto se ha comprobado que la disminución del ácido araquidónico y las prostaglandinas (PGs) constituye un componente de la teratogenicidad en la diabetes ${ }^{33,41}$.

La hiperglicemia causa disminución de los niveles de prostaglandina E2 en el embrión. Las prostaglandinas participan en funciones celulares como proliferación, diferenciación y modulación del tono vascular. Estos mediadores lipídicos son generados en la vía de la ciclooxigenasa (COX). COX es la enzima limitante en la conversión de ácido araquidónico en prostaglandina $\mathrm{H}_{2}$, que constituye el sustrato común para la síntesis de PGs.

En la gestación diabética se ha documentado la disminución de fosfoinositol 2 (PIP2), PIP3 y diacilglicerol (DAG) a consecuencia de la disminución de su precursor (inositol). PIP1 y PIP2 constituyen precursores de fosfolípidos esenciales en los tejidos embrionarios, son estimulantes de la PKC, enzima que estimula la fosfolipasa A2. Una disminución en la actividad de la fosfolipasa A2 (PLA 2) conduce a disminución del ácido araquidónico libre con disminución de la producción y el metabolismo de las prostaglandinas, sobre todo PGE2 considerada la principal prostaglandina durante la embriogénesis ${ }^{42-44}$.

La hiperglicemia, induce la regulación negativa del gen COX2 (cicloxigenasa 2) en el embrión, manteniendo su expresión disminuida, lo que puede ser un evento temprano en la embriopatía diabética al conducir a la disminución de los niveles de $\mathrm{PGs} 33,45,46$.

Por otro lado COX1 y COX2 se expresan en la membrana del retículo endoplásmico y en la envoltura nuclear. Las PGs producidas vía nuclear 
ejercen su efecto en el núcleo por interacción con receptores activados por peroxisomas que pertenecen a una superfamilia de receptores hormonales nucleares identificados como PPARs. Estos modulan la transcripción de genes blanco provocando su activación o represión en respuesta a la unión de ligandos en secuencias específicas de PPARs, y se ha reconocido que este mecanismo está involucrado en procesos patológicos como la tumorigénesis, hipertrigliceridemia y la diabetes ${ }^{47,48}$.

Por otro lado los defectos congénitos fetales en la diabetes han sido frecuentemente relacionados con alteraciones morfológicas y funcionales de la placenta, principal órgano en el transporte de nutrientes de la madre al embrión.

La diabetes afecta el funcionamiento del tejido placentario. El papel de la placenta en el desarrollo anormal de los productos de madres diabéticas es muy importante. Las placentas de madres diabéticas tienen alteraciones estructurales y funcionales, son de mayor tamaño y tienen mayor cantidad de factor de crecimiento placentario (PIGF) que está relacionado con neovascularización $^{13}$. Se han observado cambios patológicos que incluyen engrosamiento significativo de la membrana basal del trofoblasto, separación de la membrana basal en capilares basales, distensión y proliferación de células endoteliales, desorganización del espacio perivascular y disminución de la superficie vascular de las vellosidades. Estos cambios son considerados factores importantes en la hipoxia fetal ${ }^{49,50}$.

También se han observado alteraciones en la expresión de genes de proteínas de unión celular

\section{REFERENCIAS}

1. Pedersen J. The Pregnant Diabetic and Her Newborn: Problems and Management. $2^{\text {nd }}$ ed. Copenhagen, Munksgaard. 1977.

2. Wentzel P, Wentzel CR, Gareskog MB, Eriksson UJ. Induction of embryonic dysmorphogenesis by high glucose concentration, disturbed inositol metabolism, and inhibited protein kinase $\mathrm{C}$ activity. Teratology 2001; 63: 193-201.

3. Hawthorne G, SNodgrass A, Tunbridge M. Outcome of vascular ${ }^{13}$. La inducción de la diabetes modifica la expresión de los genes b laminina y fibronectina en la placenta de ratas diabéticas; cambios similares han sido demostrados en la placenta de la mujer diabética a término, lo que conduce a una alteración en la adhesión celular a este nivel que impide el normal funcionamiento del tejido placentario $^{50-53}$. Los niveles elevados de ET1 (endotelin 1) y PGE2, potentes constrictores vasculares en la placenta de diabética, se relacionan con un estado de insuficiencia placentaria e hipoxia fetal ${ }^{54}$.

Estrategias en la prevención de la embriopatía diabética. Los defectos congénitos en la gestación diabética constituyen un importante problema de salud a pesar de que se dedican numerosos esfuerzos para normalizar el riesgo de la descendencia $^{55}$. La etiología del efecto teratogénico de la diabetes no está completamente dilucidada aún pero las malformaciones congénitas producidas por diabetes experimental son prevenidas en animales con la administración de antioxidantes in vivo como a-tocopherol ${ }^{56-58}$ ascorbato $^{59}$, ácido fólico ${ }^{58}$.

En la actualidad las estrategias propuestas para la prevención de los defectos incluyen suplementación con moléculas que están deficientes en el embrión expuesto a la hiperglicemia unido a un adecuado control glicémico periconcepcional y la administración de antioxidantes para contrarrestar los efectos adversos del estrés oxidativo ${ }^{60}$. Aunque se requieren aún investigaciones adicionales, estos hallazgos corroboran el papel del metabolismo oxidativo alterado en la dismorfogénesis y sugieren la dirección para el tratamiento profiláctico futuro en humanos.

diabetic pregnancy and glucose intolerance in pregnancy: an audit of fetal loss in Newcastle General Hospital 1977-1990. Diabetes Res Clin Pract 1994; 25: 183-90.

4. Mrus JL Malformations in infants of diabetic mothers. Teratology 1982; 25: 385-94.

5. Penney GC, Mair G, Pearson DW. Outcomes of pregnancies in women with type 1 diabetes in Scotland: a national population-based study. BJOG 2003; 110: 315-8.

6. Herrera E. Metabolic adaptations in pregnancy and their implications for the availability of substrates to the fetos. Eur J Clin Nutr 2000; 54: S47-S51. 
7. LOEKEN MR. Free radicals and birth defects. J Matern Fetal Neonatal Med 2004; 15: 6-14.

8. Phelan SA, Ito M, LoekEn MR. Neural tube defects in embryos of diabetic mice: Role of the Pax3 gene and apoptosis. Diabetes 1997; 46: 1189-97.

9. Calciu C, Kubow S, Chan HM. Interactive dysmorphogenic effects of toxaphene or toxaphene congeners and hyperglycemia on cultured whole rat embryos during organogenesis. Toxicology 2002; 175: 153-65.

10. Cederberg J. Oxidative stress, antioxidative defence and outcome of gestation in experimental diabetic pregnancy. Uppsala. ISBN 2001; 91-554-4960-3.

11. Herrera E, Alvarez JJ. Cambios en las gestantes y sus implicaciones hormonales. En: Bioquímica Clínica. Madrid. Mc Graw-Hill Interamericana 1998; 345-52.

12. Goldman AS, Baker L, Piddington R, Marx B, Herold $\mathrm{R}$, EgLeR J. Hyperglicemia-induced teratogenesis is mediated by a functional deficiency of arachidonic acid. Proc Natl Acad Sci USA 1985; 82: 8227-31.

13. Polanco AC, Revilia MC, Palomino MA, Andrade S. Effect of maternal diabetes on human and rat fetal development. Ginecol Obstet Mex 2005; 73: 544-52.

14. ERICKSSON UJ, Cederberg J, Wentzel P. Congenital malformations in offspring of diabetic mothersanimal and human studies. Endocrine \& Metabolic Disorders 2003; 4: 79-93.

15. Chang TI, Horal M, Jain SK, Wang F, Patel R, Loeken MR. Oxidant regulation of gene expression and neural tube development: Insights gained from diabetic pregnancy on molecular causes of neural tube efects. Diabetología 2003; 46: 538-45.

16. Li R, THORENS B, LOEKEN MR. Expression of the gene encoding the high-K (m) glucose transporter 2 by the early postimplantation mouse embryo is essential for neural tube defects associated with diabetic embryopathy. Diabetologia 2007; 50: 682-9.

17. Wentzel P, Ejdesjö A, ERIKsson UJ. Maternal diabetes in vivo and high glucose in vitro diminish GAPDH activity in rat embryos. Diabetes 2003; 52: 1222-8.

18. BAYNES JW. Role of oxidative stress in development of complications in diabetes. Diabetes 1991; 40: 405-12.

19. Li R, Chase M, Jung SK, Smith JS, Loeken MR. Hypoxic stress in diabetic pregnancy contributes to impaired embryo gene expression and defective development by inducing oxidative stress. Am J Physiol Endocrinol Metab 2005; 289: E591-9.

20. Forsberg H, Borg LAH, Cagliero E, Eriksson UJ. Altered levels of scavenging enzymes in embryos subjected to a diabetic environment. Free Radic Res 1996; 24: 451-9.

21. ERIKSSON UJ, Borg LAH. Protection by free oxygen radical scavenging enzymes against glucose-induced embryonic malformations in vitro. Diabetologia 1991; 34: 325-31.

22. ERIKSSON UJ, Borg LAH, HagaY Z, GRONER Y. Increased superoxide dismutase (SOD) activity in embryos of transgenic mice protects from the teratogenic effects of a diabetic environment. Diabetes 1993; 42 (Suppl 1): $85 \mathrm{~A}$.

23. ERIKSSON UJ, SimA'N CM. Pregnant diabetic rats fed the antioxidant butylated hydroxytoluene show decreased occurrence of malformations in the offspring. Diabetes 1996; 45: 1497-502.

24. Vlassara H, Palace MR. Diabetes and advanced glycation endproducts. Journal of Internal Medicine 2002; 251: 87-101.

25. LoENKe MR. Advances in understanding the molecular causes of diabetes-induced birth defects. J Soc Gynecol Investig 2006; 13: 2-10.

26. Cai J, Phelan SA, Hill AL, Loeken MR. Identification of Dep-1, a new gene regulated by the transcription factor Pax3, as a marker for altered embryonic gene expression during diabetic pregnancy. Diabetes 1998; 47: 1803-5.

27. Dröge W. Free Radicals in the Physiological Control of Cell Function. Physiol Rev 2002; 82: 47-95.

28. Akazawa S. Diabetic embryopathy: studies using a rat embryo culture system and an animal model. Congenit Anom (Kyoto) 2005; 45: 73-9.

29. LOEKEN MR, HORAL M. Regulation of transcription and morphogenesis by glucosamine: does hexosamine flux mediate the molecular effects of high glucose metabolism on embryogenesis? Diabetes 2000; 49 (Suppl 1): A274.

30. Cederberg J, Gaui J, Luthman H, Ericksson UJ. Increased mRNA levels of $\mathrm{Mn}-\mathrm{SOD}$ and catalase in embryos of diabetic rats from a malformationresistant strain. Diabetes 2000; 49: 101-7.

31. Gareskog M, ERiksson UJ, Wentzel P. Combined supplementation of folic acid and vitamin E diminishes diabetes-induced embryotoxicity in rats. Birth Defects Research 2006; 76: 483-90.

32. El-Bassiouni EA, Helmy MH, Abou Rawash N, ELZoghby SM, Kamel MA, ABOU RaYAH AN. Embryopathy in experimental diabetic gestation: assessment of oxidative stress and antioxidant defence. Br J Bioed Sci 2005; 62: 71-6.

33. WentZel P, Welsh N, ERICKSSON UL. Developmental damage, increased lipid peroxidation, diminished cyclooxygenase-2 gene expression, and lowered PGE2 levels in rat embryos exposed to a diabetic environment. Diabetes 1999; 48: 813-20.

34. Sun F, Kawasaki E, Akazawa S, Hishikawa Y, Sugahara K, KaminiRa S ET aL. Apoptosis and its pathway in early post-implantation embryos of diabetic rats. Diabetes Res Clin Pract 2005; 67: 110-8.

35. Ho FM, Lin WW, Chao CM, Yang CR, Lin LI, Lai CC ET AL. High glucose-induced apoptosis in human vascular endothelial cells is mediated through NFkappaB and c-Jun NH2-terminal kinase pathway and prevented by PI3K/Akt/eNOS pathway. Cell Signal 2006; 18: 391-9. 
36. KIDD VJ. Proteolytic activities that mediate apoptosis. Annu Rev Physiol 1998; 60: 533-73.

37. Earnshaw WC, Martins LM, Kaufmann SH. Mammalian Caspases: Structure, Activation, Substrates and functions during Apoptosis. Annu Rev Bichem 1999; 68: 383-424.

38. MetCalfe AD, Hunter HR, Bloor DJ. Expression of 11 members of the BCL-2 family of apoptosis regulatory molecules during human preimplantation embryo development and fragmentation. Mol Reprod 2004; 68: 35-50.

39. Gareskog M, Wentzel P. Altered Protein Kinase C Activation Associated with Rat Embryonic Dysmorphogenesis. Pediatric Research 2004; 56: 849-57.

40. Gareskog M, Wentzel P. N-Acetylcysteine and aalpha\}cyano-4-hydroxycinnamic acid alter protein kinase C (PKC)-\{delta\} and PKC-\{zeta\} and diminish dysmorphogenesis in rat embryos cultured with high glucose in vitro. J Endocrinol 2007; 192: 207-14.

41. Eungton SK. Effects of excess glucose on mammalian post-implantation embryos. Int J Dev Biol 1997; 41: 299-306.

42. Wentzel P, Wentzel CR, Gareskog MB, Ericksson UJ. Induction of embryonic dysmorphogenesis by high glucose concentration, disturbed inositol metabolism, and inhibited protein kinase $\mathrm{C}$ activity. Teratology 2001; 63: 193-201.

43. Hiramatsu $Y$, Sekiguchi N, Hayashi M, Isshiki K, Yokota T, KING GL, LoEKen MR. Diacylglycerol production and protein kinase $\mathrm{C}$ activity are increased in a mouse model of diabetic embryopathy. Diabetes 2002; 51: 2804-10.

44. Goldman AS, Baker L, Piddington R, Marx B, Herold R, EgIER J. Hyperglicemia-induced teratogénesis is mediated by a functional deficiency of arachidonic acid. Proc Natl Acad Sci USA 1985; 82: 8227-31.

45. Wentzel P, Welsh N, ERICKsson UL. Developmental damage, increased lipid peroxidation, diminished cyclooxygenase-2 gene expression, and lowered PGE2 levels in rat embryos exposed to a diabetic environment. Diabetes 1999; 48: 813-20.

46. Lim H, Gupta AR, Wen-Ge MA, Paria BC, Moler DE, MorRow JD, DuBois RN. Cyclo-oxygenase-2-derived prostacyclin mediates embryo implantation in the mouse diabetes. Genes Dev 1999; 13: 1561-74.

47. Meade EA, McIntyre TM, Zimmerman GA, Prescott SM. Peroxisome Proliferators Enhance Cyclooxygenase-2 Expression in Epithelial Cells. J Biol Chem 1999; 274: 8328-34.

48. Higa R, González E, Pustovrh MC, White V, Capobianco E, Martínez N, Jawerbaum A. PPARd and its activator $\mathrm{PGI}_{2}$ are reduced in diabetic embryopathy: involvement of PPAR activation in lipid metabolic and signalling pathways in rat embryo early organogenesis. Molecular Human Reproduction 2007; 13, 2: 103-10.

49. Pietryga M, Biczysko W, Wender-Ozegowska E, BraZERT J, BIEGANSKA E, BICZYSKO R. Ultrastructural examination of the placenta in pregnancy complicated by diabetes mellitus. Ginekol Pol 2004; 75: 111-8.

50. Cagliero E, Forsberg H, Sala R, Lorenzi M, Eriksson UJ. Maternal diabetes induces increased expression of extracellular matrix components in rat embryos. Diabetes 1993; 42: 975-80.

51. Pedchenko VK, Chetyrkin SV, Chuang P, Ham AJ, Saleem MA, Mathieson PW et al. Mechanism of perturbation of integrin-mediated cell-matrix interactions by reactive carbonyl compounds and its implication for pathogenesis of diabetic nephropathy. Diabetes 2005; 54: 2952-60.

52. Beltramo E, Pomero F, Awone A, D’Alu F, Ponte E, PoRTA M. Pericyte adhesion is impaired on extracellular matrix produced by endothelial cells in high hexose concentrations. Diabetologia 2002; 45: 416-9.

53. Ericksson UJ, Cederberg J, Wentzel P. Congenital malformations in offspring of diabetic mothersAnimal and human studies. Endocrine \& Metabolic Disorders 2003; 4: 79-93.

54. HoRNBERGER LK. Maternal diabetes and the fetal heart. Heart 2006; 92: 1019-21.

55. Wentzel P, ERIKSSON UJ. Antioxidants diminish developmental damage induced by high glucose and cyclooxygenase inhibitors in rat embryos in vitro. Diabetes 1998; 47: 677-84.

56. SimAN CM, ERIKSSON UJ. Vitamin E decreases the occurrence of malformations in the offspring of diabetic rats. Diabetes 1997; 46: 1054-61.

57. Simán C, GitTenberger-De Groot A, Wisse B, Eriksson UJ. Malformations in offspring of diabetic rats: Morphometric analysis of neural crest-derived organs and effects of maternal vitamin $\mathrm{E}$ treatment. Teratology 2000; 61: 355-67.

58. WentZel P, ERIKSSON UJ. A diabetes-like environment increases malformation rate and diminishes prostaglandin $\mathrm{E}(2)$ in rat embryos: reversal by administration of vitamin E and folic acid. Birth Defects Res A Clin Mol Teratol 2005; 73: 506-11.

59. SimAN CM, ERIKSSON UJ. Vitamin C supplementation of the matemal diet reduces the rate of malformation in the offspring of diabetic rats. Diabetologia 1997; 40: 1416-24.

60. Cederberg J, ERIKSSON UJ. Birth Defects Research Part A: Clinical and Molecular Teratology. Antioxidative treatment of pregnant diabetic rats diminishes embryonic dysmorphogenesis. 2006; 76: 483-90. 